\title{
Rotura espontánea del tendón aquíleo
}

\section{Spontaneous Achilles Tendon Rupture}

Paciente varón de 62 años con antecedentes de extabaquismo, hipertensión arterial, dislipemia, arteriopatía periférica, hepatopatía etilica y EPOC grave que ingresa por infección respiratoria de vías aéreas bajas. Previamente había sufrido varias exacerbaciones, aislándose en cultivo de esputo Pseudomonas aeruginosa sensible a quinolonas. Al ingreso se indica biterapia con levofloxacino y piperacilina-tazobactam, esteroides y broncodilatadores. Dos semanas más tarde presenta dolor de inicio súbito en talón izquierdo, sin traumatismo ni esfuerzo previo. Al examen físico destaca equimosis y edema en región aquílea (Figura 1), con limitación para la flexión plantar por dolor. Con sospecha de rotura del tendón de Aquiles es valorado por Traumatología. Se desestima tratamiento quirúrgico por comorbilidad, indicando inmovilización mediante bota de yeso en equino con evolución posterior favorable en el plazo de tres meses.

La rotura del tendón de Aquiles se debe habitualmente a una causa traumática y ocurre en la mayoría de los casos tras una actividad física vigorosa. En ocasiones puede producirse en pacientes no ejercitados previamente, de edad más avanzada y con la práctica de determinados deportes que impliquen movimientos bruscos. Fuera de estas situaciones la rotura espontánea se produce en pacientes ancianos, con predominio en el sexo masculino y asociado a una enfermedad sistémica de base (insuficiencia renal crónica, diabetes, gota, hiperparatiroidismo, enfermedades del tejido conectivo) 0 a tratamiento esteroideo crónico. Las fluoroquinolonas son antibióticos seguros y en general bien tolerados que se relacionan raramente con tendinopatía, estimándose una incidencia de rotura tendinosa de 12/100.000 pacientes tratados ${ }^{1}$. Se desconoce el mecanismo fisiopatológico. La localización más frecuente es el tendón de Aquiles, representando el 95\% de los casos de tendinitis, con afectación bilateral hasta en el $27 \%$. El período de latencia para el desarrollo de la tendinopatía es variable, desde la primera semana hasta meses después de la retirada del fármaco, aunque la mayoría de los pacientes (85\%) presenta síntomas durante el primer mes². La supresión del fármaco más allá de la reparación quirúrgica o el manejo conservador con inmovilización y rehabilitación, constituye el tratamiento de elección.

\section{Bibliografía}

1. Corrao G, Zambon A, Bertù L, Mauri A, Paleari V, Rossi C et al. Evidence of tendinitis provoked by fluoroquinolone treatment: a case-control study. Drug Saf. 2006; 29: 889.

2. Akali AU, Niranjan NS. Manajement of bilateral Achilles tendon rupture associated with ciprofloxacin: a review and case presentation. J Plast Reconstr Aesthet Surg 2008; 61: 830.

\section{Diagnóstico}

Rotura espontánea del tendón aquíleo.

\section{Ferreira-González, JP Arequipa-Benavides.}

Servicio de Medicina Interna. Hospital Arquitecto Marcide. SERGAS. Ferrol
Figura 1. Equímosis por rotura de tendón aquíleo

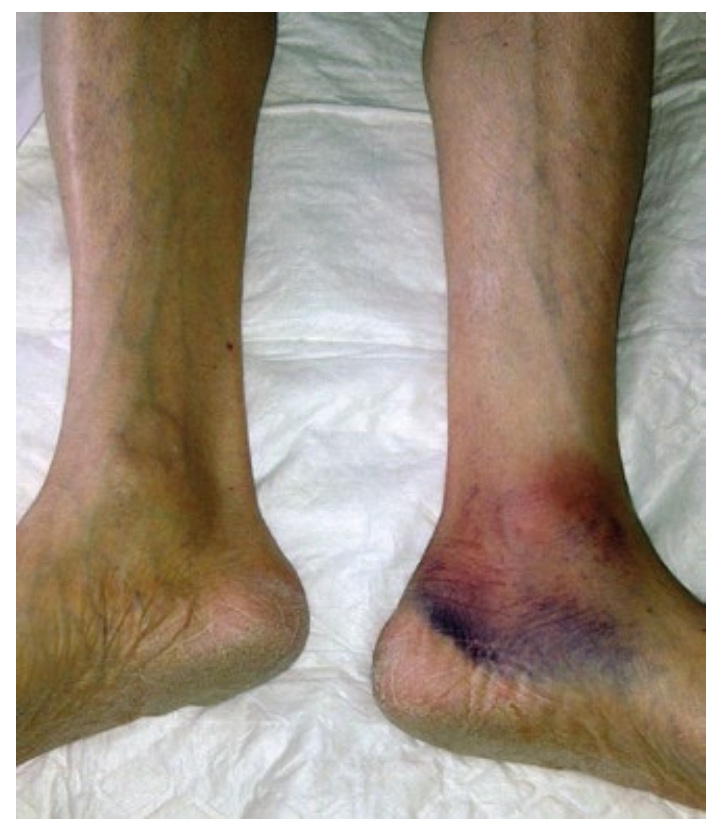

\section{Fe de erratas}

En el volumen $74, n^{\circ} 2$, dentro de las Comunicaciones Poster al XXX Congreso de la Sociedad Gallega de Medicina Interna, en la página 86 , donde figura:

EPOC Y ASPERGILLUS EN EL ÁREA DE BARBANZA.

Balo-Araujo S, Naveiro-Soneira JJ, Molinos-Castro S, García- Suarez MF, Rodríguez-Framil M, Padín-Paz EM, Varela-García PM, Diaz-Peromingo JA, Pérez Del Molino M, Iglesias-Gallego M.

H. BARBANZA, COMPLEJO HOSPITALARIO SANTIAGO

debe figurar

EPOC Y ASPERGILLUS EN EL ÁREA DE BARBANZA.

Pesqueira-Fontán PM, Rodríguez-Fernández S, Gayol-Fernández MC, Balo-Araujo S, Naveiro-Soneira JJ, Molinos-Castro S, García- Suarez MF, Rodríguez-Framil M, Padín-Paz EM, Varela-García PM, Diaz-Peromingo JA, Pérez Del Molino M, Iglesias-Gallego M.

H. BARBANZA, COMPLEJO HOSPITALARIO SANTIAGO 